\title{
BMJ Open Factors influencing psychological, social and health outcomes after major burn injuries in adults: cohort study protocol
}

\author{
Martha Druery, ${ }^{1}$ Peter A Newcombe, ${ }^{2}$ Cate M Cameron, ${ }^{3}$ Jeffrey Lipman ${ }^{1}$
}

To cite: Druery M, Newcombe PA, Cameron CM, et al. Factors influencing psychological, social and health outcomes after major burn injuries in adults: cohort study protocol. BMJ Open 2017;7:e017545. doi:10.1136/ bmjopen-2017-017545

- Prepublication history for this paper is available online. To view these files please visit the journal online (http://dx.doi org/10.1136/bmjopen-2017017545).

Received 28 April 2017 Accepted 2 May 2017

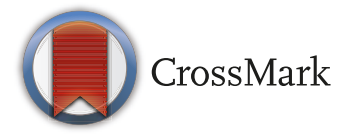

${ }^{1}$ Burns Trauma and Critical Care Research Centre, University of Queensland, Herston, Australia ${ }^{2}$ School of Psychology, University of Queensland, St Lucia, Australia

${ }^{3}$ Menzies Health Institute Queensland, Griffith University, Meadowbrook, Australia

Correspondence to

Ms. Martha Druery; m.druery@ uq.edu.au

\begin{abstract}
Introduction The goal of burn care is that 'the quality of the outcome must be worth the pain of survival'. More research is needed to understand how best to deliver care for patients with burns to achieve this aim. Loss of independence, function as well as loss of income for patients with burns and carers cause a significant burden at both individual and societal levels. Much is being done to advance knowledge in the clinical care field; however, there has been a paucity of research exploring psychosocial outcomes. This paper describes the study background and methods, as implemented in an Australian cohort study of psychosocial outcomes after major burn injuries.
\end{abstract}

Methods and analysis In this inception cohort study, a target sample of 230 participants, aged 18 years or over, admitted to a single statewide burns centre with a burn injury are identified by hospital staff for inclusion. Baseline survey data are collected either in person or by telephone within 28 days of the injury and participants then followed up with telephone interviews at 3, 6 and 12 months postburn. Injury and burns treatment information is collected from medical records. Social support is measured as a predictor variable using the Multidimensional Scale of Perceived Social Support. Outcome data are collected via standardised measures in the domains of Quality of Life (SF-12, EQ-5D, BSHS-B), depression (PHQ-9), post-traumatic stress disorder (PCL-C, PAS), community integration (CIQ-R) and Quality-Adjusted Life Years (EQ-5D). Additional survey questions measure life satisfaction, return to work and public services utilisation at 12 months postinjury. Data analysis methods will include analysis of variance, Pearson correlation and hierarchical multiple regression analyses.

Ethics and dissemination Hospital-based and University of Queensland Human Research Ethics Committees have approved the protocol. Results from the study will be disseminated at national and international conferences, in peer-reviewed journals and in a doctoral thesis.

Trial registration number Australia New Zealand Clinical Trials Registry (ACTRN12616000828426). Retrospectively registered on 23 June 2016; pre-results.

\section{INTRODUCTION}

Identifying determinants and barriers to acceptable and optimal burns outcomes is central to developing targeted interventions, programs and models of care. ${ }^{12}$
Strengths and limitations of this study

- This is a longitudinal, prospective study that incorporates injury, treatment, personal and social factors to determine predictors of longterm psychological, social and health outcomes of hospitalised adults postburn in an Australian context. As such, it will address a number of the methodological limitations that have constrained previous burns outcomes research.

- The methodology of researcher-led follow-up is intended to limit attrition, resulting in high continuing participation rates.

- The target of a larger sample size than previously reported in similar studies, in combination with multiple validated measures, will enhance the rigour of this research.

- Participants of the proposed study are patients of one Australian burns unit and therefore the results may not be generalisable to other Australian burns survivors or those from other countries.

- A further limitation is the observational nature of the study. However, observational data are sufficient to address the key research objective of reporting the psychological, social and health outcomes of the study group at 12 months postburn.

Cost-effectiveness of clinical treatment is also an important consideration due to the significant health expenditure on resource intensive burn care. It is widely agreed in the burns literature that outcome is multidimensional for burns survivors given the spectrum of impacts this type of injury has on people, physically, emotionally, psychologically and socially. $^{13}$

While a number of studies consistently report that a large proportion of burns survivors make a satisfactory functional recovery, there remain difficulties with other aspects of injury adjustment and a percentage of patients demonstrating poorer outcomes. These findings led to concentrated investigations into more global outcomes research.

Broader outcomes studies have investigated Quality of Life (QoL), ${ }^{45}$ Health-Related QoL 
(HRQoL), ${ }^{67}$ life satisfaction ${ }^{8-10}$ and service utilisation. ${ }^{11} 12$ There exists a broad spectrum of factors which have been investigated in terms of their predictive or causal relationship with burns outcomes such as QoL and HRQoL. The overall consensus is that while injury severity is strongly predictive of poorer outcome in terms of functional independence and QoL, the role of psychological and environmental factors has been empirically acknowledged. The few studies that have applied a multifactorial theoretical model to investigate the hierarchical association and direction of causality between these separate factor groupings ${ }^{46}$ have been significantly constrained by methodological limitations such as retrospective, cross-sectional designs, small sample size and one time point of data collection nearly 10 years postinjury.

The present research takes a multidimensional view. It investigates a potential range of factors, hypothesised to predict physical, psychological and health outcomes in functional, mental health, social and life satisfaction domains. The broader societal impacts of return to work, public services utilisation and 'Quality Adjusted Life Years' (QALYs) health utility weights will also be investigated.

The aim of this study is to identify factors that are predictive of psychosocial outcomes 12 months after a burn injury in order to determine which hospitalised adults are likely to experience poor long-term outcomes postburn.

\section{METHODS AND ANALYSIS}

\section{Study design}

This study uses a single-centre prospective longitudinal cohort design to capture the reported dynamic nature of burn recovery over time. ${ }^{13}$ Baseline data are collected as soon as feasible after the burn injury to gather preinjury information. Intermediate data collection occurs at 3 months postinjury (when the majority of patients will have experienced the exposure and impacts of returning to the community) and 6 months postinjury (when the acute period of care is usually completed and a rehabilitation routine has been established). Outcome data are collected at 12 months postburn.

\section{Study setting}

Participants are recruited from the Professor Stuart Pegg Adult Burn Centre at the Royal Brisbane and Women's Hospital in Australia, the sole statewide specialist adult burns referral centre for Queensland, northern New South Wales, the Northern Territory and the Pacific Islands. An average of 390 patients with a new burn injury are admitted to this burns unit annually.

\section{Study population}

\section{Inclusion criteria}

Eligible participants are those who have been admitted for at least 24 hours as an initial hospitalisation to the Professor Stuart Pegg Adult Burns Unit at the Royal Brisbane and Women's Hospital. They have either sustained a major burn injury defined as affecting $\geq 10 \%$ of the total body surface area (unless affecting the hands/forearms, feet/lower legs, head/face or perineum), the presence of airway burn or an inhalation injury, a length of stay $>7$ days or any burn injury requiring specialist multidisciplinary care in a burn centre, such as an operative procedure. To be eligible, participants are aged 18 years or over in order to legally provide consent for their own participation. Participants who are non-English speaking are also invited to participate, as this potentially marginalised group has previously been excluded from burns outcomes research in Australia. ${ }^{714}$

\section{Exclusion criteria}

The exclusion criteria includes patients who lack the requisite medicolegal capacity to provide consent to participate, those who are admitted for subsequent treatment of a previously sustained injury, not domiciled in Australia, not expected to remain so for 12 months postinjury or those who are currently incarcerated in a correctional facility. Patients with burns who are identified by the burns treating team to be experiencing significant physical or psychological symptoms are excluded as are patients who are identified to be exhibiting aggressive or violent behaviour. Patients with burns who were experiencing significant functional impairment prior to their burn injury, resulting in a high level of dependence on residential care services due to advanced age or disability are also ineligible.

\section{Sampling, recruitment and consent}

Eligible hospitalised participants in an adult burns unit are identified by nursing staff who introduce the study, advise patients of their eligibility, provide a Participant Information and Consent Form and seek agreement for a researcher to attend the unit for further discussion. Recruitment commenced in October 2016 and is ongoing. Eligibility of agreeing participants is confirmed by a member of the clinical team in relation to cognitive and psychological capacity and the researcher attends the burns unit at a clinically appropriate time. The researcher then verbally explains the study, discusses the Participant Information and Consent Form, answers questions and seeks consent. Patients who are physically able to sign the form, do so with a witness present to also sign.

\section{Data collection}

Researchers conduct an 8-minute baseline interview with consenting participants and access injury data from medical records and the hospital Burns Database. Researchers then conduct follow-up telephone interviews at 3,6 and 12 months postdate of injury. The duration of interviews are approximately 20 minutes for time points 2 and 3 and 30 minutes for time point 4 .

Data regarding injury, treatment, personal and social factors are recorded at baseline and outcomes at the follow-up time points as reported in table 1 . 
Table 1 Data collected at baseline and follow-up

Injury data $\quad$ total body surface area burned, \% full thickness burn,
circumstances, mechanism, bodily location, respiratory injury,
multitrauma
multitrauma

$\begin{array}{ll}\begin{array}{l}\text { Preburn personal and } \\ \text { social factors }\end{array} & \begin{array}{l}\text { Age, gender, relationship status, employment status, income, } \\ \text { education, remoteness of residence, housing, history of } \\ \text { trauma, prior substance use, material resources, physical and } \\ \text { psychological comorbidities, ethnicity, primary language } \\ \text { Previous health status-EQ-5D }\end{array}\end{array}$

Previous health status-EQ-5D ${ }^{25}$

Mobility, self-care, usual activities,

Social support-MSPSS ${ }^{16}$ pain/discomfort, anxiety/depression

Family, friends, significant other, global support

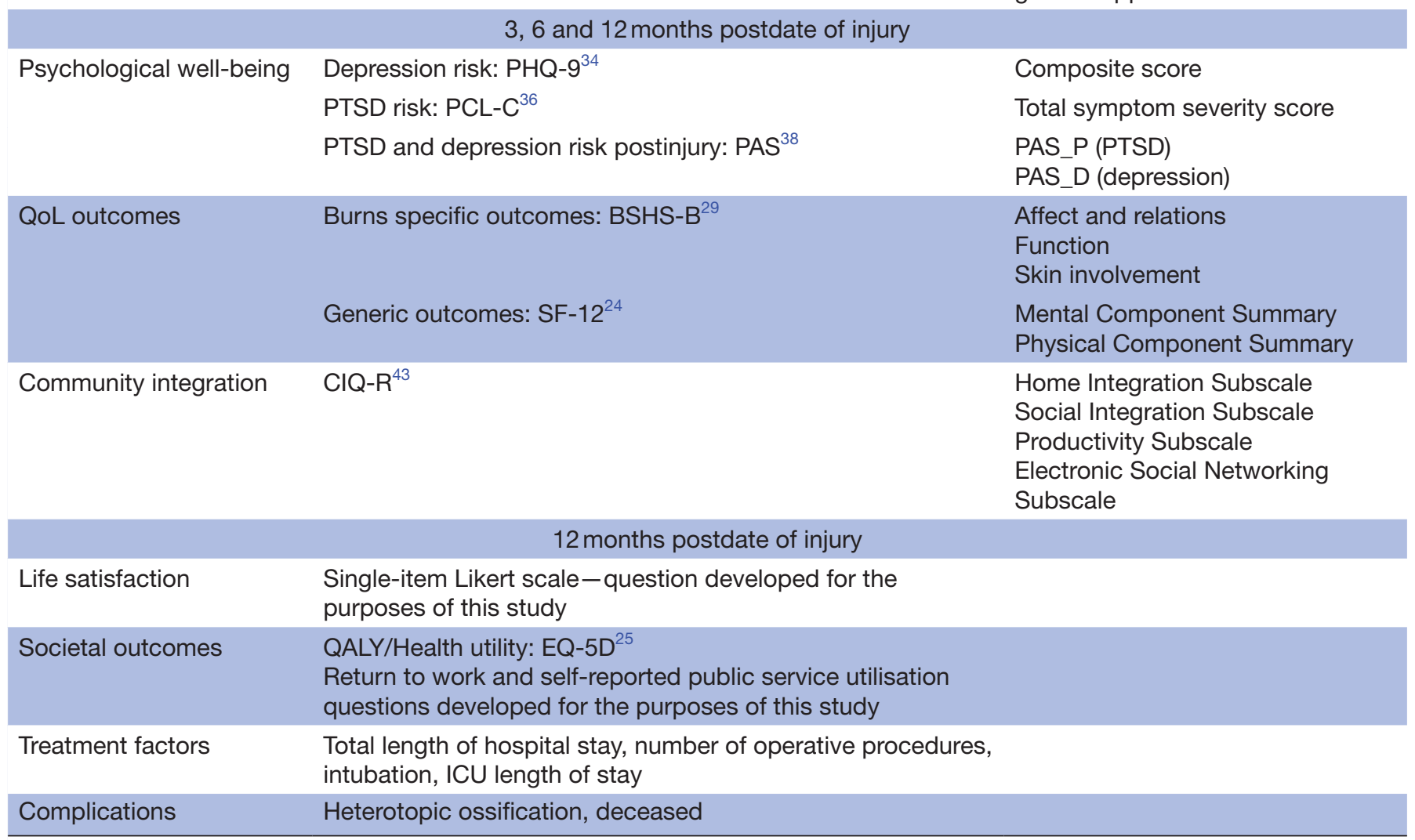

BSHS-B, Burn Specific Health Scale-Brief version; CIQ-R, revised Community Integration Questionnaire-Revised; EQ-5D, EuroQol Group Standardised Measure of Health Status; ICU, intensive care unit; MSPSS, Multidimensional Scale of Perceived Social Support; PAS, Posttraumatic Adjustment Scale; PCL-C, PTSD Symptom Checklist-Civilian version; PHQ-9, Patient Health Questionnaire; PTSD, post-traumatic stress disorder; QALY, Quality Adjusted Life Years; QoL, Quality of Life; SF-12, 12-Item Short Form Health Survey.

\section{Research measures}

Independent predictor variables

\section{Predisposing risk factors}

Premorbid individual factor data including age, gender and ethnicity are sourced from the Hospital Based Clinical Information System (HBCIS). Questions relating to premorbid use of tobacco, alcohol and recreational drugs and comorbidities have been replicated from New Zealand injury-outcome research, based on census questions. ${ }^{15}$ Premorbid social factor data such as marital status and place of residence are also sourced from HBCIS. Remoteness of residence is determined from the Australian Bureau of Statistics postcode classifications.
Questions relating to income, employment, education, housing tenure, material resources, trauma and violence history are derived from previous research. ${ }^{15}$

\section{Social support}

Due to the significance of social support as a predictor of postburn functional outcomes, ${ }^{6}{ }^{11}$ it is important to investigate in relation to psychosocial outcomes. The Multidimensional Scale of Perceived Social Support (MSPSS) is a 12-item self-report instrument which measures interpersonal functioning and social support from family, friends and significant other on a 7-point Likert scale from 'Very strongly agree' to 'Very strongly 
disagree' ${ }^{16}$ A global support score can be derived from all three subscales. Good internal reliability and validity have been reported ${ }^{1617}$ and although not validated for a burns population, it has been used extensively among mental health, injury and general populations. ${ }^{18}$

\section{Burns factors}

Injury factors including percentage of total body surface area burned, percentage of full thickness burn, circumstances of injury, mechanism of injury, visible location of injury, presence of inhalation injury and of heterotopic ossification are sourced from the Royal Brisbane and Women's Hospital burns unit database with assistance from the database manager. Treatment factors including length of stay, number of operative procedures, ventilation and intensive care unit length of stay are also sourced from the hospital burns unit database as this information is routinely collected for all admitted patients with burns.

\section{Outcome variables}

\section{Quality of Life and life satisfaction}

There is no consensus in the literature in relation to a definition or consistent measurement tool for QoL. There is also confusion whether the many component variables of QoL should be considered determinants or indicators. QoL has been defined by the WHO as 'a person's perception of his/her position in life within the context of the culture and value systems in which he/she lives and in relation to his/her goals, expectations, standards, and concerns' (WHOQOL Group, p28). ${ }^{19}$ This term has been adopted in injury outcomes literature to conceptualise the multidimensional nature of recovery across physical, functional, psychological and social domains from the patient's perspective.

There is consensus in the general injury literature to include both generic QoL and condition-specific outcome measures in study designs. ${ }^{20}{ }^{21}$ The benefit of generic outcome measurement is that it allows comparisons with normative, other injury and disease groups. ${ }^{20}$ Outcome measurement in the present study was informed by the most frequently used generic QoL tools in burns research $^{22}$; the 36-Item Short Form Health Survey (SF-36) and EuroQol Group Standardised Measure of Health Status (EQ-5D).

While the SF-36 has been validated for use in an Australian burns population, ${ }^{23}$ the SF-12 was preferred for use in the current study due to its comparable psychometric properties ${ }^{24}$ combined with its brevity, thereby reducing participant burden for this repeat measures design. This instrument includes 12 items from the original SF-36, scored to provide two summary scores: a Mental Component Summary and a Physical Component Summary. ${ }^{24}$

The EQ-5D was developed by the EuroQol Group as a generic self-report measure of health status and for economic appraisal. ${ }^{25}$ It comprises two components. The first is a descriptive system which includes responses of 'No problems', 'Some problems' or 'Extreme problems' to the five dimensions of mobility, self-care, usual activities, pain/discomfort and anxiety/depression. The second component is a Visual Analogue Scale where the participants rate their health on a given day on a vertical scale ranging from $0=$ 'Best imaginable health state' to $100=$ 'Worst imaginable health state'. The EQ-5D is recommended for use by the Cologne Group in relation to QoL outcome measurement for traumatic injuries ${ }^{26}$ and has been chosen for its sound psychometric properties and extensive use throughout the world, ${ }^{18}$ including burns outcomes research. ${ }^{2728}$

Condition-specific QoL measurement is particularly informative due to the individual consequences of burn injury that would not otherwise be captured by generic tools in isolation. ${ }^{29}$ The 40 -item Burn Specific Health ScaleBrief version (BSHS-B) has been the most frequently used measure of burns outcome in recent decades. ${ }^{30}$ The BSHS-B has been validated to measure burns outcomes in the domains of simple abilities, hand function, heat sensitivity, treatment regimens, body image, affect, interpersonal relationships, sexuality and work with excellent internal consistency reported as ranging between 0.75 and $0.93{ }^{23}$ Willebrand and Kildal ${ }^{31}$ validated a simplification of these nine domains into three clusters: affect and relations (affect, interpersonal relationships and sexuality), function (simple abilities, hand function) and skin involvement (heat sensitivity, treatment regimens and body image). These authors treated the work subscale as a separate domain due to an identified issue of double loadings.

In addition to these, concept analyses have found that subjective satisfaction with life is also of merit. ${ }^{32}$ Therefore, a single question referring to overall life satisfaction has been included with a 5-point Likert scale response, ranging from 1 (Extremely dissatisfied with my life in general at the moment) to 5 (Extremely satisfied with my life in general at the moment).

\section{Psychological well-being}

The domains of mental health that are recommended for routine outcome measurement by the American Burn Association (ABA) are depression and post-traumatic stress disorder. ${ }^{33}$ They recommend the Patient Health Questionnaire (PHQ-9) ${ }^{34}$ for screening of depression in the inpatient or outpatient adult burns population. The PHQ-9 is a 9-item self-report measure which has been adapted for assessing depression severity from the Primary Care Evaluation of Mental Disorders instrument (PRIME-MD). ${ }^{35}$ The items are scored from 0 (Not at all) to 3 (Nearly every day), providing a composite score from 0 to 27 and lower-bound cut-off points for depression severity levels of none (0), mild (5), moderate (10), moderately severe (15) and severe (20). ${ }^{34}$ The internal reliability of the PHQ-9 has been reported to be excellent $(\alpha=0.89)$ with similarly sound sensitivity, specificity and construct validity. ${ }^{35}$

The ABA recommends the Post-Traumatic Stress Disorder (PTSD) Symptom Checklist—Civilian version (PCL-C) for assessing PTSD if assessment by clinical interview is not feasible. ${ }^{33}$ These authors report that the 
PCL-C is also routinely used in American traumatic brain injury and spinal cord injury populations. It is a self-report measure, derived for civilian use from a military tool, with 5-point Likert scale responses $(1=$ 'Not at all', $5=$ 'Extremely') to 17 items. ${ }^{36}$ Its internal consistency, test-retest reliability and validity have been reported to be good. ${ }^{37}$ A total symptom severity score, ranging from 17 to 85 , can be determined by summing the scores. Ruggiero and colleagues ${ }^{37}$ determined that a cut-off score of 44 is sufficient for identifying PTSD.

An additional predictive screening index for both PTSD and depression, the Post-traumatic Adjustment Scale (PAS), has specifically been developed for administration following traumatic injury. ${ }^{38}$ This 10 -item scale yields a summary PAS_P score indicating risk for PTSD (at 16 or greater) and a summary PAS_D score for depression (at 4 or above) with good psychometric properties. Its inclusion in the current study will allow the potential for validation in a burns population, not previously published.

\section{Community integration}

Areas of community integration such as social, recreational and vocational activities have been found to be predictive of psychosocial outcomes in burns survivors ${ }^{13} 39$ as well as investigated as an important outcome measure postburn. ${ }^{40}$ Originally a measure designed to assess productive activity, home competency and social integration in acquired brain injury patients, the Community Integration Questionnaire $(\mathrm{CIQ})^{41}$ has been recommended for use with adult patients with burns. ${ }^{1}$ It has not been validated for use with the burns population but has been extensively used in other studies due to its sound psychometric properties. ${ }^{42}$ The CIQ was revised in 2014 (CIQ-R) to include an 'evaluating social networking' (ESN) subscale, scoring improvements and Australian population normative data. ${ }^{43}$ It is an 18 -item self-report instrument which measures participation in the operation of the home, activities outside the home, interpersonal relations, involvement in employment, education and volunteer activities and participation in ESN. The original CIQ author, Willer, recommends use of the improved CIQ-R. ${ }^{43}$

\section{Return to work}

As a key component of functional outcome postburn, ${ }^{27} 44$ employment status information will be collected at 12 months postinjury. For those who were employed at the time of burn, data will be collected regarding the number of weeks between burn injury and return to work. Information will also be collected in relation to whether the participant has resumed normal hours and activities or reduced arrangements.

\section{Public service utilisation}

Questions with binary 'Yes/No' answers will be included in the survey booklet at 12 months postburn in relation to usage of non-burns related health services, mental health services, alcohol and drug services, public rental housing, Centrelink income support and corrective services.
Free-text fields will be used to collect comments in relation to the nature of these services.

\section{Health utility}

The EQ-5D, which is used as a generic QoL outcome measure also provides a health utility outcome/QALY measure. Each participant's health state is derived from scoring the five descriptive system domains into a 5-digit code. This code can then be converted into a single summary index with a formula that results in a QALY for each participant.

\section{Sample size}

Online calculation using Epi Info online software (https://wwwn.cdc.gov/epiinfo/7/index.htm) yielded a target sample size of 138 completions at 12 months postburn. Input values for the sample size calculation were based on $\alpha$ of $5 \%$, a standard power of $90 \%$, with a large effect size and incidence or exposure estimates as follows. The expected incidence of poor psychosocial outcomes in the burns population with good social resources is $20 \%$ and for those with poor social resources is $50 \%$, based on reported figures in the OECD Better Life Index (https:/ / www.oecdbetterlifeindex.org) and conventional calculations from injury and disease epidemiology literature, with a ratio of exposed to unexposed participants estimated at 2.5. Allowing for $40 \%$ attrition, which approximates that reported in the burns literature, ${ }^{4546}$ a final sample size of 230 is required for statistical significance.

\section{Data analysis}

Summary statistics will be used to describe the study cohort. Categorical data will be expressed as frequencies and percentages. A missing values analysis will be conducted to determine the randomness of the missing data. The outcome of these analyses will guide the appropriate missing value replacement method (eg, multiple imputation, linear interpolation) as required to ensure statistical power is maintained at $90 \%$. The statistical methods will include analysis of variance to explore changes across time points and Pearson correlation to explore relationships between risk factors, burns factors and social support with psychological, social and health outcomes. Hierarchical multiple regression analyses will be used to test the predictability of injury, treatment, social and environmental factors on burns outcomes, adjusted for potentially confounding covariates (eg, premorbid individual factors). All statistical analyses will be two-tailed and considered significant at $\mathrm{p}<0.05$. Free-text data collected in relation to public service utilisation will be subject to thematic analysis for descriptive reporting of response categories.

\section{DISCUSSION}

The primary focus of this study is to examine associations and predictors for long-term psychological, social and health outcomes for hospitalised patients with burns encompassing life satisfaction, psychological well-being, 
functional outcomes, health status, return to work and community integration. It is hypothesised that preburn social factors such as low income, unemployment, remoteness of residence, poor housing tenure, limited material resources and restricted social support will be significant predictors of poorer psychosocial outcomes in adult survivors with burns. It is also hypothesised that limited preburn social resources will be significantly associated with poorer health utility weights and greater public service utilisation at 12 months postinjury.

Burns studies have used various time points for prospective longitudinal data collection but few have provided a rationale for the choice of schedule. The US National Institute on Disability and Rehabilitation Research Burn Model System Database is a large repository of burn injury incidence, treatment and outcome data which has designated follow-up outcome data to be collected at the time of discharge then at 6, 12 and 24 months after injury. ${ }^{2}$ These time points are similar to those propounded by the Trauma Registry of the American College of Surgeons at time of discharge, 1 month, 6 months and 12 months postburn. ${ }^{33}$ The Cologne Group, a multinational consensus forum regarding QoL outcome measurement of trauma survivors, also recommends data collection at T0 (preinjury), T1 (acute phase-3 months postinjury), T2 (rehab phase-6 months) and T3 (long-term outcome phase-12 months). ${ }^{26}$ These time points represent phases of recovery calculated from date of injury as opposed to date of discharge, which was abandoned by the European Consumer Safety Association working group on injury-related outcomes measurement guidelines ${ }^{21}$ due to variability. It has been shown that there is little change after 12 months postburn, ${ }^{47}$ a finding echoed in the general injury literature, ${ }^{48} 49$ so this has been chosen as an end-point for outcome data collection in the present study. Although the practice of retrospectively collecting patient-reported preinjury data is prone to recall bias, ${ }^{49}$ it is important to determine the preburn situation and circumstances of participants. ${ }^{1}{ }^{50}$ Data are not collected at the time of discharge as this is a variable time point dependent on injury severity, complications and other factors. The schedule will remain static for all participants for consistency of analysis.

An economic analysis of costs associated with injury also supports this methodology given that the major costs associated with long-term, static sequelae eclipse the high cost of medical and rehabilitative treatment in the acute and initial improvement phases. ${ }^{48}$

There is a risk that survivors with burns who consent to participate in the study will differ from those who decline, which will introduce bias. Comparison of participants and non-participants will be possible in relation to routinely collected hospital data for all admitted patients with burns. Attrition is an added risk for any longitudinal study design, introducing potential constraints in generalisability of findings from the study cohort to the burns population. However, it will be possible to assess any differences in those who become lost to follow-up over the course of the project.
Recall bias is limited due to the prospective design of the proposed study; however, this will be a potential source of error in relation to baseline measurement of premorbid individual and social factors as well as health status. An attempt to minimise this is made by collecting baseline data as soon as possible after admission to hospital or after resumption of cognitive capacity. The measures invite participants to recall varying periods of time from 'at the moment' to 'in the last month'. While this is also a potential source of bias, it has been reported not to be a significant difficulty for respondents. ${ }^{51}$

Participants of the proposed study are patients of one Australian burns unit and therefore the results may not be generalisable to other Australian burns survivors or those from other countries. However, the Queensland population is largely representative of the nation, ${ }^{52}$ so the relationships between factors and outcomes as well as direction of effects are likely to be similar in other regions. As cognitively impaired burns survivors are excluded due to the limitations inherent in proxy reporting, results will not be generalisable to those with cognitive deficits. The study will also only recruit survivors with burns who were admitted for their burn injury. Those who did not require treatment in the statewide burns centre or hospitalisation may also be significantly affected by their burn injury but not be represented by this sampling method..$^{53}$

An added limitation is due to the decision not to include a measure of cognitive functioning. Although other researchers have extended the EQ-5D to measure this, ${ }^{21}{ }^{54}$ the amendment arguably undermines the tool's psychometric properties. Measurement of cognitive functioning is beyond the scope of the present study which is focused on the potential relationship between preinjury social factors and psychosocial outcomes.

A further limitation is the risk of response bias or Hawthorne Effect due to the interview modality of administering the surveys, which is inherent in self-report measures. It would be optimal to integrate self-report responses with clinician assessments or observations; however, feasibility of the study precluded this option.

This study addresses a number of the methodological limitations that has constrained previous research as it is a longitudinal, prospective study that incorporates injury, treatment, personal and social factors to determine predictors of long-term psychological, social and health outcomes of hospitalised adults postburn in an Australian context. The methodology of researcher-led follow-up is intended to limit attrition and the anticipated high participation rate will optimise recruitment of a representative sample for generalisable findings. The target of a larger sample size than previously reported in similar studies, in combination with multiple validated measures, contribute to the rigour of this research.

The findings will provide detailed information about the multiple impacts experienced over time by survivors of major burn injuries in the study population. It will reveal factors that are associated with the highest risk of adversity and the greatest societal costs. The results are 
anticipated to assist in identifying those patients with burns who are more likely to experience poorer psychosocial outcomes, in order that evidence-based intervention, services and programs can be delivered to target these more vulnerable people. Identifying factors influencing injury outcomes is intended to lead to future research on modifiable factors for better outcomes in the population after serious burn-injury and cost-effective improvements.

\section{Ethics and dissemination}

The study protocol has been approved by the Royal Brisbane and Women's Hospital Human Research Ethics Committee (HREC/15/QRBW/145) and the Behavioural Social Sciences Ethics Review Committee, University of Queensland (2015001089). The appropriate permissions have been granted for access and usage of all specified databases, clinical information systems, patient records and standardised measures. Results from this study will be disseminated at national and international conferences, in peer-reviewed journals and in a doctoral thesis.

Correction notice This paper has been amended since it was published Online First. Owing to a scripting error, some of the publisher names in the references were replaced with 'BMJ Publishing Group'. This only affected the full text version, not the PDF. We have since corrected these errors and the correct publishers have been inserted into the references.

Acknowledgements The authors thank Michael Rudd and Anne Webster, the Director and the Nurse Unit Manager, respectively, of the Professor Stuart Pegg Adult Burn Centre as well as Angela 0'Malia, Director of Social Work and Psychology and multidisciplinary inpatient and outpatient burn care teams at the Royal Brisbane and Women's Hospital for their support in the research. The assistance of the Burns Unit Database Manager, Teresa Matthews, research assistants, Narelle Simmons and Claire Simpson and social work students, Lia Burton, Christopher Hampson and Joseph Toohey, with participant recruitment and data collection are also gratefully acknowledged.

Contributors MD conceived of the study and its design, drafted the grant proposal, manages its coordination as the principal investigator of the study and drafted the manuscript. PAN and CMC participated in the design of the study and all authors, including $\mathrm{JL}$, critically revised the manuscript and approved the final version of the manuscript.

Funding Thisstudy was funded by a Royal Brisbane and Women's Hospital Foundation Grant andthrough the generous support of Red Blanket Miracle. MD was supported by anAustralian Post-Graduate Award and a RBWH Professor Stuart Pegg AdultBurns Centre Scholarship.

\section{Competing interests None declared.}

Ethics approval The study protocol has been approved by the Royal Brisbane and Women's Hospital Human Research Ethics Committee (HREC/15/QRBW/145) and the Behavioural Social Sciences Ethics Review Committee, University of Queensland (2015001089).

\section{Provenance and peer review Not commissioned; peer reviewed.}

Data sharing statement This article does not contain any data. No datasets have been analysed during the current phase of the study; therefore, data sharing is not applicable. Study materials and future datasets may be available from the corresponding author on reasonable request.

Open Access This is an Open Access article distributed in accordance with the Creative Commons Attribution Non Commercial (CC BY-NC 4.0) license, which permits others to distribute, remix, adapt, build upon this work non-commercially, and license their derivative works on different terms, provided the original work is properly cited and the use is non-commercial. See: http://creativecommons.org/ licenses/by-nc/4.0/

(C) Article author(s) (or their employer(s) unless otherwise stated in the text of the article) 2017. All rights reserved. No commercial use is permitted unless otherwise expressly granted.
REFERENCES

1. Falder S, Browne A, Edgar D, et al. Core outcomes for adult burn survivors: a clinical overview. Burns 2009;35:618-41.

2. Klein MB, Lezotte DL, Fauerbach JA, et al. The National Institute on Disability and Rehabilitation Research burn model system database: a tool for the multicenter study of the outcome of burn injury. $J$ Burn Care Res 2007;28:84-96.

3. Palmieri TL, Przkora R, Meyer WJ, et al. Measuring burn injury outcomes. Surg Clin North Am 2014;94:909-16.

4. Kildal M, Andersson G, Gerdin B. Health status in Swedish burn patients. Assessment utilising three variants of the Burn Specific Health Scale. Burns 2002;28:639-45.

5. Ying WL, Pertrini MA, Xin LL. Gender differences in the quality of life and coping patterns after discharge in patients recovering from burns in China. Journal of Research in Nursing 2013;18:247-62.

6. Moi AL, Wentzel-Larsen T, Salemark L, et al. Long-term risk factors for impaired burn-specific health and unemployment in patients with thermal injury. Burns 2007;33:37-45.

7. Wasiak J, Lee SJ, Paul E, et al. Predictors of health status and health-related quality of life 12 months after severe burn. Burns 2014;40:568-74.

8. Costa BA, Engrav LH, Holavanahalli R, et al. Impairment after Burns: a two-center, prospective report. Burns 2003;29:671-5.

9. Goverman J, Mathews K, Nadler D, et al. Satisfaction with life after burn: a Burn Model System National Database Study. Burns 2016;42:1067-73.

10. Patterson DR, Ptacek JT, Cromes F, et al. The 2000 clinical research award. describing and predicting distress and satisfaction with life for burn survivors. J Burn Care Rehabil 2000;21:490-8.

11. Liang CY, Wang HJ, Yao KP, et al. Predictors of health-care needs in discharged burn patients. Burns 2012;38:172-9.

12. Wikehult $B$, Willebrand M, Kildal M, et al. Use of healthcare a long time after severe burn injury; relation to perceived health and personality characteristics. Disabil Rehabil 2005;27:863-70.

13. Cromes GF, Holavanahalli R, Kowalske K, et al. Predictors of quality of life as measured by the Burn Specific Health Scale in persons with Major burn injury. J Burn Care Rehabil 2002;23:229-34.

14. Wasiak J, Paul E, Lee SJ, et al. Patterns of recovery over 12 months following a burn injury in Australia. Injury 2014;45:1459-64.

15. Langley J, Derrett S, Davie G, et al. A cohort study of short-term functional outcomes following injury: the role of pre-injury sociodemographic and health characteristics, injury and injury-related healthcare. Health Qual Life Outcomes 2011;9:68.

16. Zimet GD, Powell SS, Farley GK, et al. Psychometric characteristics of the Multidimensional Scale of perceived Social support. J Pers Assess 1990;55:610-7.

17. Zimet GD, Dahlem NW, Zimet SG, et al. The Multidimensional Scale of perceived Social support. J Pers Assess 1988;52:30-41.

18. Kenardy J, Heron-Delaney M, Bellamy N, et al. The University of Queensland study of physical and psychological outcomes for claimants with minor and moderate injuries following a road traffic crash (UQ SuPPORT): design and methods. Eur J Psychotraumatol 2014;5:22612.

19. WHOQOL Group. Development of the WHOQOL: rationale and current status. Int J Ment Health 1994;23:24-56.

20. van Baar ME, Essink-Bot ML, Oen IM, et al. Functional outcome after Burns: a review. Burns 2006;32:1-9.

21. Van Beeck EF, Larsen CF, Lyons RA, et al. Guidelines for the conduction of follow-up studies measuring injury-related disability. $J$ Trauma 2007;62:534-50.

22. Meirte J, van Loey NE, Maertens $\mathrm{K}$, et al. Classification of quality of life subscales within the ICF framework in burn research: identifying overlaps and gaps. Burns 2014:40:1353-9.

23. Edgar D, Dawson A, Hankey G, et al. Demonstration of the validity of the SF-36 for measurement of the temporal recovery of quality of life outcomes in burns survivors. Burns 2010;36:1013-20.

24. Ware J, Kosinski M, Keller SD. A 12-Item Short-Form Health survey: construction of scales and preliminary tests of reliability and validity. Med Care 1996;34:220-33.

25. EuroQol Group. EuroQol--a new facility for the measurement of health-related quality of life. Health Policy 1990;16:199-208.

26. Bouillon B, Kreder HJ, Eypasch E, et al. MI Consensus Group. Quality of life in patients with multiple injuries--basic issues, assessment, and recommendations. Restor Neurol Neurosci 2002;20:125-34.

27. Orwelius L, Willebrand M, Gerdin B, et al. Long term health-related quality of life after burns is strongly dependent on pre-existing disease and psychosocial issues and less due to the burn itself. Burns 2013;39:229-35. 
28. Pavoni V, Gianesello L, Paparella L, et al. Outcome predictors and quality of life of severe burn patients admitted to intensive care unit. Scand J Trauma Resusc Emerg Med 2010;18:24.

29. Kildal M, Andersson G, Fugl-Meyer AR, et al. Development of a brief version of the Burn Specific Health Scale (BSHS-B). J Trauma 2001;51:740-6.

30. Wasiak J, McMahon M, Danilla S, et al. Measuring common outcome measures and their concepts using the International classification of functioning, disability and Health (ICF) in adults with burn injury: a systematic review. Burns 2011;37:913-24.

31. Willebrand M, Kildal M. A simplified domain structure of the burnspecific health scale-brief (BSHS-B): a tool to improve its value in routine clinical work. J Trauma 2008;64:1581-6.

32. Moons P, Budts W, De Geest S. Critique on the conceptualisation of quality of life: a review and evaluation of different conceptual approaches. Int J Nurs Stud 2006;43:891-901.

33. Gibran NS, Wiechman S, Meyer W, et al. American Burn Association Consensus Statements. Journal of Burn Care \& Research 2013;34:361-85.

34. Kroenke K, Spitzer RL. The PHQ-9: a New Depression Diagnostic and Severity measure. Psychiatr Ann 2002;32:509-15.

35. Kroenke K, Spitzer RL, Williams JB. The PHQ-9: validity of a brief depression severity measure. J Gen Intern Med 2001;16:606-13.

36. Blanchard EB, Jones-Alexander J, Buckley TC, et al. Psychometric properties of the PTSD checklist (PCL). Behav Res Ther 1996;34:669-73.

37. Ruggiero KJ, Del Ben K, Scotti JR, et al. Psychometric properties of the PTSD Checklist-Civilian version. J Trauma Stress 2003;16:495-502.

38. O'Donnell ML, Creamer MC, Parslow R, et al. A predictive screening index for posttraumatic stress disorder and depression following traumatic injury. J Consult Clin Psychol 2008;76:923-32.

39. Cobb N, Maxwell G, Silverstein P. Patient perception of quality of life after burn injury. results of an eleven-year survey. J Burn Care Rehabil 1990;11:330-3.

40. Esselman PC, Ptacek JT, Kowalske K, et al. Community integration after burn injuries. J Burn Care Rehabil 2001;22:221-7.
41. Willer B, Rosenthal M, Kreutzer JS, et al. Assessment of community integration following rehabilitation for traumatic brain injury. $J$ Head Trauma Rehabil 1993;8:75-87.

42. Sander AM, Fuchs KL, High WM, et al. The Community integration questionnaire revisited: an assessment of factor structure and validity. Arch Phys Med Rehabil 1999;80:1303-8.

43. Callaway $L$, Winkler $D$, Tippett $A$, et al. The Community integration questionnaire - Revised (CIQ-R). Melbourne: Summer Foundation Ltd, 2014.

44. Mason ST, Esselman P, Fraser R, et al. Return to work after burn injury: a systematic review. J Burn Care Res 2012;33:101-9.

45. Fauerbach JA, Lawrence J, Stevens S, et al. Work status and attrition from longitudinal studies are influenced by psychiatric disorder. $J$ Burn Care Rehabil 1998;19:247-52.

46. Holavanahalli RK, Lezotte DC, Hayes MP, et al. Profile of patients lost to follow-up in the Burn Injury Rehabilitation Model Systems longitudinal database. J Burn Care Res 2006;27:703-12.

47. Ullrich PM, Askay SW, Patterson DR. Pain, depression, and physical functioning following burn injury. Rehabil Psychol 2009;54:211-6.

48. Baldry Currens JA, Coats TJ. The timing of disability measurements following injury. Injury 2000;31:93-8.

49. Polinder S, Haagsma JA, Belt E, et al. A systematic review of studies measuring health-related quality of life of general injury populations. BMC Public Health 2010;10:783.

50. Edgar D, McMahon M, Plaza A, et al. In: Edgar D, ed. Burn Trauma Rehabilitation: allied Health Practice guidelines. Philadelphia: Lippincott Williams \& Wilkins, 2014:84-99.

51. Harcombe H, Derrett $S$, Herbison P, et al. "Do I really want to do this?" Longitudinal cohort study participants' perspectives on postal survey design: a qualitative study. BMC Med Res Methodol 2011;11:8.

52. Office of Economic and Statistical Research. QTa T, ed. Census 2011: Queensland's Population Compared. Queensland: Queensland Government, 2012.

53. Kahn SA, Bell DE, Hutchins P, et al. Outpatient burn data: an untapped resource. Burns 2013;39:1351-4.

54. Krabbe PF, Stouthard ME, Essink-Bot ML, et al. The effect of adding a cognitive dimension to the EuroQol multiattribute health-status classification system. J Clin Epidemiol 1999;52:293-301. 